\title{
Parameters derived from integrated nuclear fluorescence, syntactic structure analysis, and vascularization in human lung carcinomas
}

\author{
Klaus Kayser, ${ }^{\mathrm{a}, *}$, Barbara Richter ${ }^{\mathrm{a}}$, Richard Stryciak ${ }^{\mathrm{a}}$ and Hans-Joachim Gabius ${ }^{\mathrm{b}}$ \\ a Abteilung für Pathologie, Thoraxklinik, 69126 Heidelberg, Germany \\ ${ }^{\mathrm{b}}$ Institut für Physiologische Chemie, Tierärztliche Fakultät, Ludwig-Maximilians-Universität, \\ 80539 München, Germany
}

\begin{abstract}
Combined measurements of integrated nuclear fluorescence (INF) and vascularization were performed on surgical specimens of human lung carcinomas. Histological slides of formalin-fixed, paraffin-embedded tissue samples were treated with Texas Red-labeled antibody to factor VIII and the fluorochrome DAPI. The resulting images were analyzed with an epiillumination fluorescence microscope and two different filter blocks. The first image displayed the vessels, and the second the DAPI-stained nuclei of surrounding cells. The extent of vascularization was assessed by calculating the volume fraction $(\mathrm{Vv})$, the surface fraction (Sv), the area, and the minimum diameter of the vessels. The INF was measured in tumour cells and lymphocytes, and was grouped according to the distance from the nearest vascular boundary into the intervals of $0-20$, 21-40, 41-60, 61-80, and $>80 \mu$. The numerical densities (Nv) as well as the percentages of S-phase-related tumour cell fraction (SPRF) and of tumour cells with an INF $>5 \mathrm{C}$ were computed. A minimum of 50 vessels and 300 tumour cells were examined. The material included 100 cases with primary lung carcinoma (39 epidermoid carcinomas, 39 adenocarcinomas, 13 large cell carcinomas, three small cell anaplastic carcinomas, and 6 carcinoid tumours). On the average, the volume density of the stroma amounts to $16.7 \%$, and that of the vessels ( $\mathrm{Vv}$ ) to $12.8 \%$. The minimum diameter of the intratumoral vessels is $13 \mu$ and the measured circumference $138 \mu$. The numerical densities of tumour cells (lymphocytes) decrease with increasing distance from the vascular boundary from $6.3(1.7)$ to $1.0(0.1)$. A reduction is also seen in the percentage of the SPRF from 10.7 to $8.1 \%$. The percentage of tumour cells with an INF $>5 \mathrm{C}$, however, is positively correlated to the distance from the vascular surfaces from 34.2 to $38.2 \%$. The measurements reveal that tumour cells are densely positioned and have an increased proportion of proliferation in the populations close to perivascular spaces, whereas chromosome abnormalities are seen more frequently, when tumour cells are located at a distance $>20 \mu$ from the vascular surfaces.
\end{abstract}

Keywords: Lung carcinoma, vascularization, integrated nuclear fluorescence, S-phase-related tumour cell fraction, numerical density

\section{Introduction}

Angiogenesis is an essential process to sustain the local expansion of tumours [8,9]. Their degree of vascularization may thus have an impact on diverse cellular properties of the tumour cell

\footnotetext{
*Mailing address: K. Kayser, Department of Pathology, Thoraxklinik, Amalienstr. 5, D-69126 Heidelberg, Germany. Tel.: +1 496221 396496; Fax: +1 496221396238.
}

0921-8912/97/\$8.00 @ 1997 - IOS Press. All rights reserved 
(sub)populations. This influence can then translate into distinct aspects of the clinical behavior of the tumour entity $[3,10,11,15,21]$. Evidently, it is plausible to assume that descriptive properties for a solid tumour such as the percentage of proliferating or aneuploid tumour cells or syntactic structure analysis-derived data sets may be correlated to the distance to the vascular boundary. Instead of a rather global monitoring of these features, segmentation can refine the definition of distinct spatial categories. If solid experimental evidence is provided for this assumption, strategies to interfere with angiogenesis can eventually have a bearing on survival rates in appropriately selected groups. At present, similar research on breast cancer has not yet enabled to come a clear-cut conclusion concerning this question $[3,5,6,18,20,22,35,36,39,45-47,51,52]$. Tumour angiogenesis has been reported to be associated with the prognosis in early stage breast carcinomas and with lymph node metastasis $[51,52]$. Similar data have been reported from melanomas, ovarian cancer, and prostatic cancer $[16,21,43,50]$. With respect to lung cancer no apparent correlation between the density of intratumoural microvessels and presence of lymph node metastases could be discerned [41], although some investigators reported an association between vascularization and tumour extent $[32,33,54]$.

In this study, we followed the above reasoning by combining assessment of the vascular density in lung tumours, the surface density and the minimum diameter of the vessels, cytometric properties of tumour cells such as degree of aneuploidy, and features of syntactic structure analysis of tumour and inflammatory host cells by stereological and segmentation techniques. The results presented here underscore that the distance between tumour cells and the vascular surface is correlated with the structural organization of tumour cell clusters and their content of proliferating and aneuploid cells.

\section{Material and methods}

\subsection{Material}

Histological slides (4-6 $\mu$ thick) were obtained from 100 surgical specimens of primary human lung tumours excised at the Thoraxklinik (Heidelberg, Germany) during the period of 01.01-30.06. 1994. Only specimens from lobes and lungs with potentially curative resection were investigated in this study. A detailed macroscopic examination including the analysis of at least one complete tumour cross-section, accurate postsurgical TNM classification, and a determination of the tumour cell type according to the WHO classification was routinely performed [53]. The material consisted of 94 carcinomas of the major cell type, and 6 rare tumours (see Table 1). The tumour cell classification was based upon HE-, PAS- and Feulgen-stained slides, and on immunohistochemical techniques, if

Table 1

Synopsis of material (number of cases and mean age)

\begin{tabular}{lrc}
\hline & N & Age (years) \\
\hline Sex & 78 & 63 \\
- males & 22 & 58 \\
- females & & \\
& & \\
Cell type & 39 & 61 \\
- epidermoid & 39 & 62 \\
- adeno & 3 & 62 \\
- small cell & 13 & 59 \\
- large cell & 6 & 62 \\
- others (carcinoid, mesothelioma) & 6 & \\
\hline
\end{tabular}


necessary. A random sectioning (nucleator) of the tumours according to the technique described by Gundersen [17] was performed. This technique permits a non-biased estimation of volumes and volume fractions of the segmented vessels. In addition, it can be used to analyze whether the vessels have a preferred orientation, i.e., are anisotropic.

\subsection{Staining}

The sections were cut from formalin-fixed, paraffin-embedded tissue blocks. After deparaffination, a part of the sections was incubated with Texas Red (sulforhodamine 101 acid chloride)-labeled monoclonal antibody against factor VIII (Dako, Hamburg, Germany) at room temperature for $30 \mathrm{~min}$. A 4',6-diamidino-2-phenylindole dihydrochloride (DAPI)-containing solution (MoBiTec, Göttingen, Germany) was applied at a concentration $15 \mathrm{ng} / \mathrm{ml}$ of phosphate-buffered saline at $40^{\circ} \mathrm{C}$ for $10 \mathrm{~min}$. After short but intensive rinsing to remove the free dye the sections were mounted in Entellan (Merck, Darmstadt, Germany).

\subsection{Technical equipment and measurements}

The stained slides were analyzed with an epi-illumination fluorescence microscope (Fluovert, Leica, Bensheim, Germany) using a $100 \mathrm{~W}$ mercury arc lamp and a NPL fluorar objective $(\times 25), 0.6$. Filter blocks TX13833 (Texas Red) and A513824 (DAPI) were used to distinguish the different fluorescence images. The distribution profiles of the dyes were captured with an one chip black/white CCD camera (Leica, Bensheim, Germany) connected to a frame grabber (Leutron XFP, Munich, Germany). The measurements were performed with an automated image-analyzing system based upon a commercially available image-analyzing system (DIAS, Towersoft, Berlin, Germany), and self-written programs. The software was installed on a $100 \mathrm{MHz}$ Pentium PC equipped with a 220 MB magnetic-optical disk. The areas of interest were selected interactively, and the images were recorded directly after staining. The measurements included an interactive segmentation of the vessels in the Texas Red image, and a semi-automatic segmentation of the nuclei in the DAPI image. The vascular walls were segmented, and expanded in 4 discrete steps. Each step increased the minimum diameter for $20 \mu$ in both directions, and the maximum diameter in respect to the relationship of the minimum/maximum diameter. The perivascular spaces were then computed by artificial expansion of the vascular boundaries in accordance to the new minimum and maximum diameter, and then the INF of those cells located within the computed area was computed. The individual steps comprised 4 expansions of additional $20 \mu$ in diameter, respectively. If a nucleus was part of two different spaces, that of the nearest vessels was taken into account. The following features were computed:

(a) vessels: minimum diameter, circumference, area, volume fraction $(\mathrm{Vv})$, surface fraction $(\mathrm{Sv})$, stroma (volume fraction of stained endothelium);

(b) nuclei: numerical density (Nv), area, S-phase-related tumour cell fraction (SPRF: $2.75<$ INF $<3.25$ ), percentage of tumour cells with an INF $>5 \mathrm{C}$, modified entropy according to Stenkvist and Starande (INF entropy) [44], surface current of entropy as described by Kayser et al. [27], vascular current of entropy (see below), the distance between nearest neighboring cells grouped into the categories of tumour cells, SPRF-cells, cells with an INF $>5 \mathrm{C}$ or lymphocytes, the structural entropy as described by Kayser (MST entropy) [27], surface current of entropy, and vascular current of entropy (see below). Calculation of distances between cell nuclei was based on the construction of the minimum spanning tree (MST), following a procedure outlined elsewhere [25-28]. A minimum number of 50 vessels and of 300 tumour cells per case was measured. Intratumorous lymphocytes served for the calculation of the $2 \mathrm{C}$ peak. 


\subsection{Comments on the calculation of features derived from cytometry}

In flow cytometry, the DNA content of nuclei can be associated with the stage of the cell cycle, and cells belonging to the S/G1 phase or with an obnormal DNA content can in general be identified. Although cytometric data derived from tissue sections are not as precise as those derived from flow or image cytometry, the contents of integrated optical densities (IOD) still reflect the cytometric "gold standard". Tumour cells with $2.75<\mathrm{IOD}<3.25$ and with an IOD $>5 \mathrm{C}$ are of specific biological importance in lung carcinoma patients. Patients suffering from lung carcinomas with a high percentage of one of these tumour cell fractions have a poor prognosis [26,28]. These tumour cell fractions have been measured on tissue sections, and are called S-phase-related fractions (SPRF) and tumour cell fraction, where INF $>5 \mathrm{C}$. The reader should be aware that the terms do not imply that the actual percentages of these fractions are measured but only estimate tumour cell fractions with a proven biological significance.

\subsection{Calculation of the vascular current of entropy}

The current of entropy is a feature which measures the distance of a thermodynamically open system from its equilibrium stage [27]. The heat produced by all energy-generating processes within a tumour has to be carried off through the surfaces of the tumour. These include the surrounding boundary (surface), and the inner surfaces (vessels). It seems, therefore, appropriate to define the current of entropy flowing through the vascular surfaces (vascular current of entropy) by the following equation:

$$
\mathrm{ECV}=\mathrm{E} \times \mathrm{SPRF} / \mathrm{Sv},
$$

$\mathrm{ECV}=$ current of entropy passing through the boundaries of the vessels, $\mathrm{E}=$ entropy, SPRF $=$ $\mathrm{S}$-phase-related tumour cell fraction, $\mathrm{Sv}=$ surface/volume fraction of the vessels. This equation was used for the computation of the currents of INF entropy and structural entropy (based upon the construction of the MST). The concept is derived from the assumption that the entropy changes are mainly related to those cells which are proliferating [27], i.e., these cells are responsible sources for entropy differences which have to be transferred through the inner surface.

\section{Results}

Synopsis of the material is given in Table 1. The average age of the patients at the time of surgical treatment and the frequency distribution of the pTN classification (Table 2) is comparable to the tumour parameters of the patients treated surgically within the last years [24,28]. The ratio of tumour volume/volume of surgical specimens is $7 \%$ on average. Therefore, more than $90 \%$ of healthy tissue had to be excised for a potentially curative resection. The features of the vascular system within the tumours are shown in Table 3. The area of the vascular cross-sections measured $1510 \mu^{2}$ on average with a minimum diameter of $13 \mu$. The volume fraction of stroma and of vessels is similar, and about $30 \%$ of the tumour volume appears to belong to non-tumourous structures. The cytometric features are summarized in Table 4. The S-phase-related fraction amounts to $10.5 \%$, as measured with the fluorescent dye, and is therefore comparable to results obtained with Feulgen-stained tissue collections $[25,28]$. The technical equivalence of the two methods is further supported by the percentage of tumour cells with an INF $>5 \mathrm{C}$ which is nearly identical to the value based on the integrated optical density 
Table 2

General features - macroscopy (mean and standard deviation)

\begin{tabular}{lcc}
\hline Feature & Mean & Standard deviation \\
\hline Tumour volume & $80 \mathrm{~cm}^{3}$ & $147 \mathrm{~cm}^{3}$ \\
Specimen volume & $1,1240 \mathrm{~cm}^{3}$ & $1,021 \mathrm{~cm}^{3}$ \\
Ratio & $7 \%$ & $10 \%$ \\
Number of excised lymph nodes/case & 7.2 & 2.5 \\
\hline Tumour stages & $\mathrm{N}$ & Mean tumour volume $\left(\mathrm{cm}^{3}\right)$ \\
\hline - pT1 & 32 & 6 \\
- pT2 & 54 & 106 \\
- pT3 & 9 & 129 \\
- pT4 & 3 & 170 \\
- pN0 & 53 & 68 \\
- pN1 & 21 & 76 \\
- pN2 & 18 & 121 \\
- pN3 & 6 & 132 \\
\hline
\end{tabular}

Table 3

General features - stereology (distance in $\mu$, area in $\mu^{2}$ )

\begin{tabular}{lrc}
\hline Feature & Mean & Standard deviation \\
\hline Vv stroma & 16.7 & 15.8 \\
Vv vessels & 12.8 & 7.7 \\
Sv vessels & 1.6 & 0.5 \\
& 1510 & 1010 \\
Area & 13 & 7 \\
Minimum diameter & 138 & 79 \\
Circumference & 95 & 82 \\
Theoretical circumference & & \\
Tc ${ }^{*}$ & \\
${ }^{*}$ Calculated according to $\left(\mathrm{Tc}=2 \pi r_{\min }\right) ; r_{\text {min }}=$ minimum vascular diameter.
\end{tabular}

Table 4

General features (cytometry)

\begin{tabular}{lcc}
\hline Feature & Mean & Standard deviation \\
\hline S-phase-related fraction & 10.5 & 2.5 \\
Percent $>$ 5C & 36.5 & 11.8 \\
2CV & 5.5 & 2.7 \\
& 1.73 & 0.16 \\
INF entropy & 7.2 & 4.9 \\
Current of entropy (surface) & 25.2 & 20.1 \\
Current of entropy (vessels) & 25.2 & \\
\hline
\end{tabular}

Table 5

General features (syntactic structure analysis, distances in $\mu$ )

\begin{tabular}{lcc}
\hline Feature & Mean & Standard deviation \\
\hline Distance between & & \\
- tumour cells & 15.8 & 2.6 \\
- proliferating tumour cells & 66.7 & 13.5 \\
- tumour cells with INF > 5C & 25.2 & 20.1 \\
- tumour cells-lymphocytes & 22.9 & 2.8 \\
& 21.5 & 10.3 \\
MST entropy & 10.7 & 8.4 \\
Current of entropy (surface) & 37.3 & 34.6 \\
Current of entropy (vessels) & 37.3 \\
\hline
\end{tabular}


Table 6

Nuclear features (mean) in relation to cell distance from the vascular surface

\begin{tabular}{lccccc}
\hline Feature & \multicolumn{5}{c}{ Distance $(\mu)$} \\
\cline { 2 - 6 } & $0-20$ & $21-40$ & $41-60$ & $61-80$ & $>80$ \\
\hline Nuclear area $\left(\mu^{2}\right)$ & $53^{*}$ & 58 & 57 & 58 & 58 \\
Tumour cell Nv & $6.3^{*}$ & 1.6 & 1.0 & 0.8 & 1.0 \\
Lymphocyte Nv & $1.7^{*}$ & 0.4 & 0.3 & 0.2 & 0.1 \\
S-phase-related fraction Nv & $0.7^{*}$ & 0.1 & 0.09 & 0.04 & 0.04 \\
INF $>$ 5C Nv & $2.3^{*}$ & 0.5 & 0.4 & 0.3 & 0.4 \\
S-phase-related fraction $(\%)$ & $10.7^{*}$ & 8.1 & 8.1 & 8.1 & 8.2 \\
Tumour cells with INF $>$ 5C & $34.2^{*}$ & 39.0 & 39.0 & 38.5 & 38.2 \\
\hline
\end{tabular}

${ }^{*}$ Statistically significant $(p<0.01)$.

\section{CELLULAR FEATURES IN RELATION TO TUMOUR CELL DISTANCE FROM VASCULAR SURFACE}

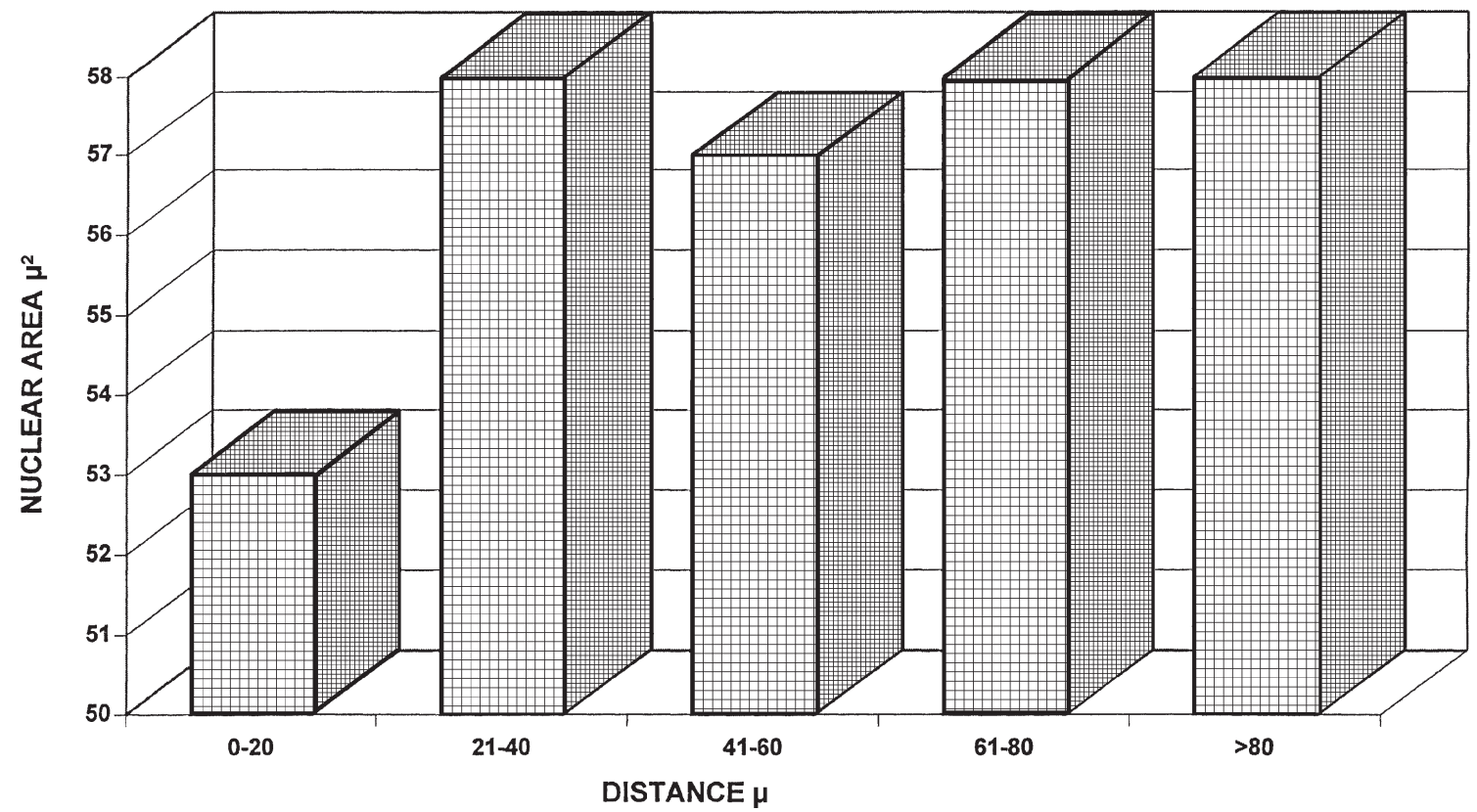

Fig. 1. Mean of nuclear area of tumour cells grouped according to their distance from the nearest vascular surface (area in $\mu^{2}$; distance in $\mu$ ).

(IOD > 5C), reported previously [28]. The various features obtained by syntactic structure analysis are presented in Table 5. It is notable that the distances between tumour cells appear to be smaller than the distances between tumour cells and neighboring lymphocytes. The amount of entropy transported through the vascular surface is markedly larger than that through the tumour boundary. This difference emphasizes the importance of the vascular system for the thermodynamical equilibrium of a malignant lung tumour. The cellular features depend upon the distance of tumour cells to the nearest vessel, as demonstrated in Table 6 and Figs 1,2. The tumour cell nuclei are smaller, the numerical density of tumour cells and lymphocytes is higher, and the relative number of proliferating tumour cells is larger, if the tumour cells are located within a distance of $20 \mu$ from the vascular boundary. 


\section{CELLULAR FEATURES IN RELATION TO TUMOUR CELL DISTANCE FROM VASCULAR SURFACE}

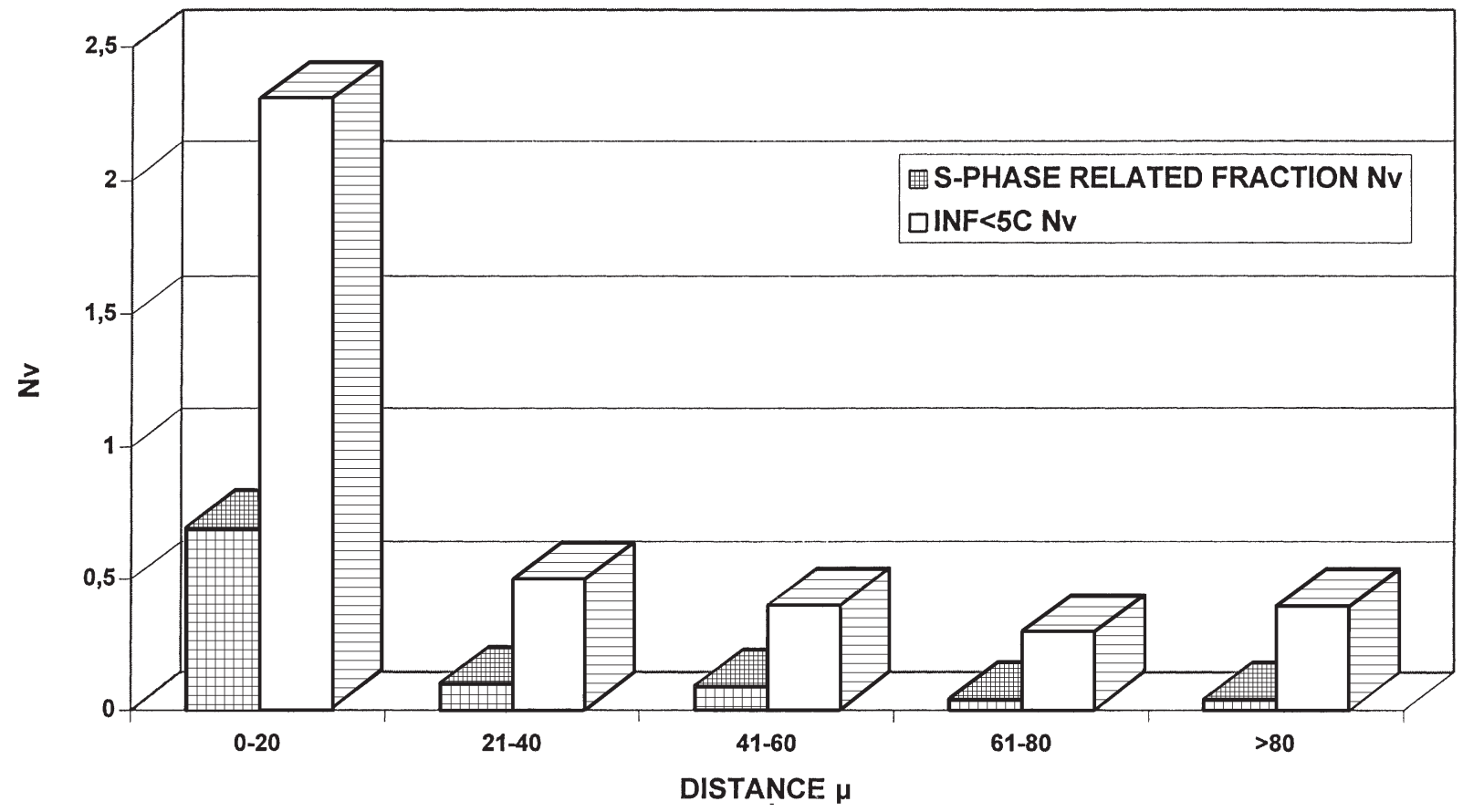

Fig. 2. Nuclear volume densities (Nv) of tumour cells with an integrated nuclear fluorescence (INF) within the S-phase-related tumour cell fraction $(2.75<\mathrm{INF}<3.25)$, and an INF $>5 \mathrm{C}$ grouped according to their distance from the nearest vascular surface (distance in $\mu$ ).

\section{Discussion}

The basic idea of this analysis is based on the assumption that the structural organization of tumour cell populations and the spatial distribution of the parameters derived from measurement of DNA content-related features (IOD, INF) is commonly heterogeneous. Since the spatial expansion and the viability of solid tumours depend on their vascularization, it is an intriguing question as which to extent the factor of spatial vicinity to vessels can have a bearing on these topological and biochemical tumour cell properties.

Form the technical point of view, the technique of quantitative fluorescence microscopy was chosen. As indicators for vessel localization and DNA content a Texas Red-labeled antibody to factor VIII and the fluorescent dye DAPI, respectively, were used. The application of the fluorochrome DAPI is fast, allowing even an intraoperative evaluation [23], and as reliable as the Feulgen stain [23,49]. Thus, it had to be proven that measurements of the integrated optical density (IOD) and of integrated nuclear fluorescence give comparable results. The prerequisite is fulfilled in this series: all cytometric data obtained from integrated fluorescence measurements are in accordance with the results of lung tumour analyses performed with Feulgen-stained slides [26-28].

The measurements of vascular density are commonly assessed by quantitation of positivity for an endothelial cell-specific marker in a predefined area, which is supposed to be representative for the complete tumour specimen $[3,5,6]$. Using a monitoring at low magnification, areas with dense 
vessel presence can preferentially be evaluated, establishing the hot-spot technique [3,5,20]. Since the pattern of growth in angiogenesis may not necessarily be isotropic, it is expedient to account for this fact by a deliberate randomization, i.e., cutting the specimen at a series of angles. Moreover, the detailed computation of volume densities, surface densities and numerical volume densities, provided by stereological approaches [17], can establish a further refinement of the current status in this field. Therefore, we used the technique of a non-biased sectioning of the surgical specimens described by Gundersen et al. [17].

The experimental series reveal that the vascular density is equivalent to approximately $15 \%$ of the tumour volume. Another $12 \%$ of the entire volume is occupied by the extracellular matrix surrounding the vessels. Their average minimum diameter is $13 \mu^{2}$, and the average length of a vascular profile is $138 \mu$. Since the average surface density exceeds the value calculated on the basis of the average minimum diameter by a factor of 1.5 , the orientation of the vessels is likely to be anisotropic, i.e., without a predefined direction. This irregular growth calls for adequate randomization of cutting angles to avoid a faulty representation.

The measurements presented here have addressed the questions as to whether the structural organization of tumour cells and/or DNA content-related features can depend on the extent of their vicinity to vessels. Indeed, parameters from both types of analysis, i.e., density of the tumour cell population and the size of the S-phase-related fraction, reveal a negative correlation with the tumour cell-vessel distance, whereas the percentage of aneuploid cells displays a positive correlation. The markedly increased cellular and numerical density of tumour cells close to the vascular surface may reflect, in addition, the interaction between endothelial cells and tumour cells [1,2,31,37].

The interpretation of the results is difficult. No similar experiment has been reported until now to our knowledge. In addition, the present lack of a precise knowledge about the molecular factors which effect this behavior and the reported heterogeneity of the endothelial cell populations $[4,7,30$, $34,38,40,42,48,55]$ notwithstanding, it is pertinent to extend such studies to the delineation of the putatively causative features, namely the expression of the defined biochemical determinants. As one graphic example, we refer to the regulation of expression of lectins, which are involved in a variety of physiological processes encompassing growth control mechanisms [12]. Distinct changes in the composition of tissue culture medium in vitro, e.g., addition of retinoic acid or phorbol ester, or alterations of a biochemically less defined nature, e.g., transfer of tumour cells to different microenvironments, lead to a marked alteration of lectin presence, quantitatively and/or qualitatively $[13,14]$. Notably, initial combined studies have previously documented that the presence of receptors for certain oligosaccarides such as histo-blood group $\mathrm{A} / \mathrm{H}$-trisaccharides is connected to parameters focused on in this report and to the survival of lung cancer patients $[24,25]$. This type of combined investigation is therefore proposed to provide a guideline to eventually disclose the identity of factors which mediate the recognitive interactions establishing clinically relevant characteristics [26,29].

Future analyses will take into consideration the influence of additional factors, especially the different tumour cell types and their interactions with the immune system. Within this framework, the influence of the vicinity to vessels deserves further attention [56]. Besides tumour cell properties, the potential functional heterogeneity of this population $[7,19,42]$ will also have to be taken into account.

\section{Acknowledgements}

The financial support of the Verein zur Förderung des biologisch-technologischen Fortschritts is gratefully acknowledged. 


\section{References}

[1] R. Auerbach, Interaction between cancer cells and the endothelium, in: The Microcirculation in Cancer Metastasis, F.W. Ort and L. Weiss, eds, CRC Press, London, 1991, pp. 169-181.

[2] R. Auerbach, J. Plendl and B. Kusha, Endothelial cell heterogeneity and differentiation, in: Angiogenesis in Health and Disease, M.E. Maragoudakis, ed., Plenum Press, New York, 1992, pp. 55-62.

[3] K. Axelsson, B.M.E. Ljung, D.H. Moore, D.H. Thor, K.L. Chew, S.M. Edgerton, H.S. Smith and B.H. Mayall, Tumour angiogenesis as a prognostic assay for invasive ductal breast carcinoma, J. Natl. Cancer Inst. 87 (1995), 997-1008.

[4] M.P. Bevilaqua, Endothelial leucocyte adhesion molecules, Ann. Rev. Immunol. 11 (1993), 767-804.

[5] S. Bosari, A.K.C. Lee, R.A. DeLellis, B. Wiley, G.J. Heatley and M.L. Silverman, Microvessel quantitation and prognosis in invasive breast carcinoma, Hum. Pathol. 23 (1992), 755-761.

[6] P. Costello, A. McCann, D.N. Carney and P.A. Dervan, Prognostic significance of microvessel density in lymph node negative breast carcinoma, Hum. Pathol. 26 (1995), 1181-1184.

[7] L.F. Fajardo, The complexity of endothelial cells, Am. J. Clin. Pathol. 92 (1989), 241-250.

[8] J. Folkman, What is evidence that tumours are angiogenesis dependent?, J. Natl. Cancer Inst. 82 (1990), 4-6.

[9] J. Folkman, K. Watson, D. Ingber and D. Hanahah, Induction of angiogenesis during the transition from hyperplasia to neoplasia, Nature 339 (1989), 58-61.

[10] G. Fontanini, D. Bigini, S. Vignati, F. Basolo, A. Murri, M. Lucchi, S. Chine, C.A. Angelett, A.L. Harris and P. Bevilaqua, Microvessel count predicts metastatic disease and survival in non-small lung cancer, J. Pathol. 177 (1995), 57-63.

[11] A.E. Fraire, V.L. Roggli, R.T. Vollmer, S.D. Greenberg, M.H. McGarvan, H.J. Spjut and R. Yesner, Lung cancer heterogeneity - prognostic implications, Cancer 60 (1987), 370-375.

[12] H.J. Gabius and S. Gabius, eds, Glycosciences: Status and Perspectives, Chapman and Hall, London, 1997.

[13] H.J. Gabius and K. Vehmeyer, Effect of microenvironment and cell-line type on carbohydrate-binding proteins of macrophage-like cells, Biochem. Cell Biol. 66 (1988), 1169-1176.

[14] S. Gabius, N. Yamazaki, W. Hanewacker and H.J. Gabius, Regulation of distribution, amount and ligand affinity of sugar receptors in human colon carcinoma cells by treatment with sodium butyrate, retinoic acid and phorbol ester, Anticancer Res. 10 (1990), 1005-1012.

[15] D. Gospodarowicz, Expression and control of vascular endothelial cells: proliferation and differentiation by fibroblast growth factors, J. Invest. Dermatol. 93 (1989), 39-45.

[16] C.H. Graham, J. Rivers and R.S. Kerbel, Extent of vascularization as a prognostic indicator in thin $(<0.76 \mathrm{~mm})$ malignant melanomas, Am. J. Pathol. 145 (1994), 510-514.

[17] H.J.G. Gundersen, T.F. Bendtsen, L. Korbo, N. Marcussen, A. Möller, K. Nielsen, J.R. Nyengaard, B. Pakkenberg, F.B. Sörensen, A. Vesterby and M.J. West, Some new, simple and efficient stereological methods and their use in pathological research and diagnosis, APMIS 96 (1988), 379-394.

[18] N.R. Hall, D.E. Fish, N. Hunt, R.D. Goldin, P.J. Guillou and J.R.T. Monson, Is the relationship between angiogenesis and metastasis in breast cancer real?, Surg. Oncol. 1 (1992), 223-229.

[19] I.R. Hart and A. Saini, Biology of tumour metastasis, Lancet 339 (1992), 1453-1458.

[20] M.E.H.M. van Hoef, W.F. Knox, S.S. Dhesi, A. Howell and A.M. Schor, Assessment of tumour vascularity as a prognostic factor in lymph node negative invasive breast cancer, Eur. J. Cancer 29A (1993), 1141-1145.

[21] H.C. Hollingsworth, E.C. Kohn, S.M. Steinberg, M.L. Rothenberg and M.J. Merino, Tumour angiogenesis in advanced stage ovarian carcinoma, Am. J. Pathol. 147 (1995), 33-41.

[22] E.R. Horak, R. Leek, N. Klenk, S. LeJeune, K. Smith, N. Stuart, M. Greenall, K. Stepniewska and A.L. Harris, Angiogenesis assessed by platelet/endothelial cell adhesion molecule antibodies, as indicator of node metastases and survival in breast cancer, Lancet 340 (1992), 1120-1124.

[23] K. Kayser, K. Baumgartner and H.J. Gabius, Cytometry with DAPI-stained tumour imprints - a reliable tool for improved intraoperative analysis of lung neoplasms, Anal. Quant. Cytol. Histol. 18 (1996), 115-120.

[24] K. Kayser, N.V. Bovin, E.Y. Korchagina, C. Zeilinger, F.Y. Zeng and H.J. Gabius, Correlation of expression of binding sites for synthetic blood group A-, B-, and H-trisaccharides and for sarcolectin with survival of patients with bronchial carcinoma, Eur. J. Cancer 30A (1994), 653-657.

[25] K. Kayser, N.V. Bovin, F.-Y. Zeng, C. Zeilinger and H.J. Gabius, Binding capacities to blood-group antigens A, B and H, DNA and MST measurements, and survival in bronchial carcinoma, Radiol. Oncol. 28 (1994), 282-286.

[26] K. Kayser, C. Kayser, W. Rahn, N.V. Bovin and H.J. Gabius, Carcinoid tumours of the lung: immuno- and ligandohistochemistry, analysis of integrated optical density, syntactic structure analysis, clinical data, and prognosis of patients treated surgically, J. Surg. Oncol. 63 (1996), 99-106. 
[27] K. Kayser, K. Kremer and M. Tacke, Integrated optical density and entropiefluss (current of entropy) in bronchial carcinoma, In Vivo 7 (1993), 387-392.

[28] K. Kayser, F. Liewald, K. Kremer, M. Tacke, M. Storck, P. Faber and P. Bonomi, Alteration of integrated optical density and intercellular structure after induction chemotherapy and survival in lung carcinoma patients, Anal. Quant. Cytol. Histol. 16 (1994), 18-24.

[29] A. Kolin, Tumour angiogenesis in human lung adenocarcinoma [letter], Cancer 75 (1995), 151.

[30] S. Kumar, D.C. West and A. Ager, Heterogeneity in endothelial cells from large vessels and microvessels, Differentiation 36 (1987), 57-70.

[31] I. Kuzu, R. Bicknell and A.L. Harris, Heterogeneity of vascular endothelial cells with relevance to diagnosis of vascular tumours, J. Clin. Pathol. 45 (1992), 143-148.

[32] P. Macchiarini, G. Fontanini, E. Dulmet, V. de Montpreville, A.R. Chapellier, J. Cerrino, F.L. Ladurie and P.G. Danteville, Angiogenesis: an indicator of metastasis in non-small cell lung cancer invading the thoracic inlet, Ann. Thorac. Surg. 57 (1994), 1534-1539.

[33] P. Macchiarini, G. Fontanini, M.J. Hardin, F. Squartini and C.A. Angeletti, Relation of neovascularization to metastasis of non-small cell lung cancer, Lancet 340 (1992), 145-146.

[34] S.A. McCarthy, I. Kuzu, K.C. Gatter and R. Bicknell, Heterogeneity of the endothelial cell and its role in organ preference and tumour metastasis, TIPS 12 (1991), 462-467.

[35] D. Milliaris, A. Kamas and H. Kalekou, Angiogenesis in invasive breast carcinoma: is it associated with parameters of prognostic significance?, Histopath. 26 (1995), 165-169.

[36] F. Monschke, W.U. Muller, U. Winkler and S. Streffers, Cell proliferation and vascularization in human breast carcinoma, Int. J. Cancer 49 (1991), 812-815.

[37] J.D. Pearson, Endothelial cell biology, Radiology 179 (1991), 9-13.

[38] W. Risau, Development of the vascular system of organs and tissues, in: Collateral Circulation, W. Schaper and J. Schaper, eds, Kluwer, Dordrecht, 1993, pp. 17-28.

[39] A.A. Sahin, N. Sneige, G.N. Ordonez, E. Singletery and A.G. Ayala, Tumour angiogenesis detected by Ulex europeus agglutinin 1 lectin (UEA1) and factor VIII immunostaining in node negative breast carcinoma (NNBC) treated by mastectomy: prediction of tumour recurrence, Mod. Pathol. 6 (1993), 19.

[40] H. Schnürch and W. Risau, Expression of tie-2, a member of a novel family of receptor tyrosine kinases, in the endothelial cell lineage, Development 119 (1993), 957-968.

[41] J. Sikora, J. Slodkowska, J. Kobos and A. Radomyski, Tumour angiogenesis within lung adenocarcinoma and ist correlation with metatstasis - application of CD34 endothelial marker, Electr. J. Pathol. Histol. $961-02$ (1996).

[42] F. Sinowatz and F. Plendl, Heterogeneity of endothelial cells, Electr. J. Pathol. Histol. 962-08 (1996).

[43] A. Srivastava, P. Laidler, R.P. Davies, K. Horgan and L.E. Hughes, The prognostic significance of tumour vascularity in intermediate-thickness (0.76-4.0 mm thick) skin melanoma, Am. J. Pathol. 133 (1988), 419-423.

[44] B. Stenkvist and G. Strande, Entropy as an algorithm for the statistical description of DNA cytometric data obtained from image analysis microscometry, J. Anal. Cell. Pathol. 2 (1990), 159-166.

[45] M. Toi, J. Kashitani and T. Tominaga, Tumour angiogenesis is an independent prognostic indicator in primary breast carcinoma, Int. J. Cancer 55 (1993), 371-374

[46] R.K. Vartanian and N. Weidner, Correlation of intratumoural endothelial cell proliferation with microvessel density (tumour angiogenesis) and tumour cell proliferation in breast carcinoma, Am. J. Pathol. 144 (1994), 1188-1194.

[47] D.W. Visscher, S. Smilanetz, S. Drozdowicz and S.M. Wyhes, Prognostic significance of image morphometric microvessel enumeration in breast carcinoma, Anal. Quant. Cytol. Histol. 15 (1993), 88-92.

[48] J.M. Wang, S. Kumar, D. Pye and A.L. Harris, A monoclonal antibody detects heterogeneity in vascular endothelium of tumours and normal tissues, Int. J. Cancer 54 (1993), 363-370.

[49] N. Wang, Y. Pan, T. Heiden and B. Tribukait, Fluorescence image cytometry for measurement of nuclear DNA content in surgical pathology, Cytometry 22 (1995), 323-329.

[50] N. Weidner, P.R. Carroll, J. Flax, W. Blumenfeld and J. Folkman, Tumour angiogenesis correlates with metastasis in invasive prostate carcinoma, Am. J. Pathol. 143 (1993), 401-409.

[51] N. Weidner, J. Folkman, F. Pozza, P. Bevilaqua, E.N. Allred, D.H. Moore, S. Meli and G. Gasparini, Tumour angiogenesis: a new significant and independent prognostic indicator in early-stage breast carcinoma, J. Natl. Cancer Inst. 84 (1992), 1875-1887.

[52] N. Weidner, J.P. Semple, W.R. Welch and J. Folkman, Tumour angiogenesis and metastasis - correlation in invasive breast carcinoma, N. Engl. J. Med. 324 (1991), 1-8.

[53] World Health Organization (WHO), Histological Typing of Lung Tumours, 2nd edn, Geneva, 1982. 
[54] K. Yamazaki, S. Abe, H. Takekawa, N. Sukoh, N. Watanabe, S. Ogura, I. Nakajima, H. Isobe, K. Inoue and Y. Kawakami, Tumour angiogenesis in human lung adenocarcinoma, Cancer 74 (1994), 2245-2250.

[55] B.R. Zetter, Endothelial heterogeneity: influence of vessel size, organ localization and species specificity on the properties of cultured endothelial cells, in: Endothelial Cells, U.S. Ryan, ed., Vol. 2, CRC Press, Boca Raton, 1989, pp. 63-79.

[56] D. Zhu, C.F. Chang and B.U. Pauli, Mediation of lung metastasis of murine melanomas by a lung-specific endothelial cell adhesion molecule, Proc. Natl. Acad. Sci. USA 88 (1991), 9568-9572. 


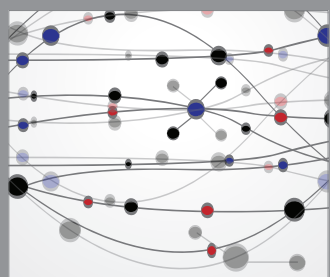

The Scientific World Journal
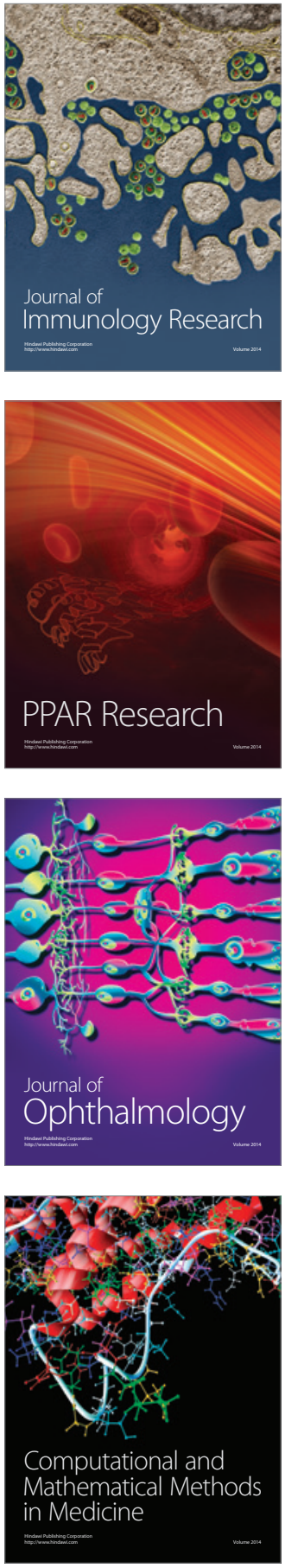

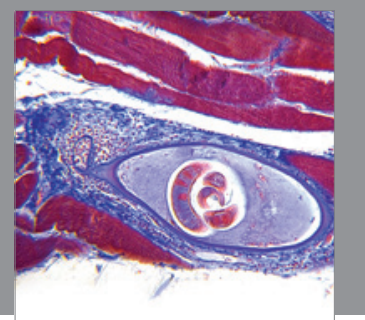

Gastroenterology

Research and Practice
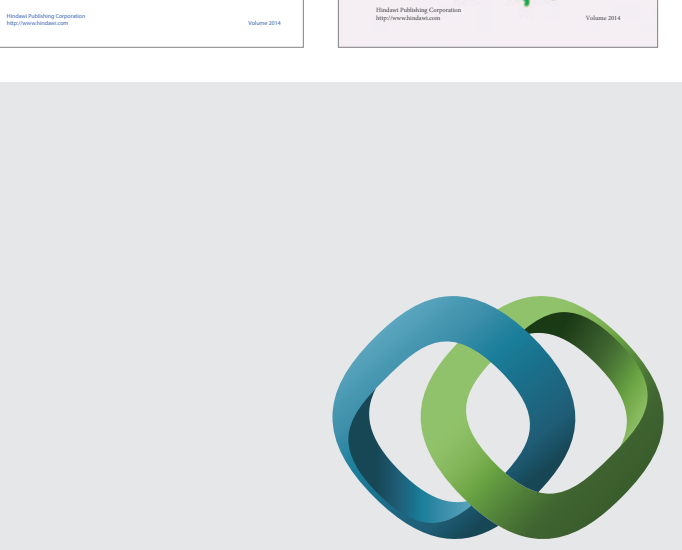

\section{Hindawi}

Submit your manuscripts at

http://www.hindawi.com
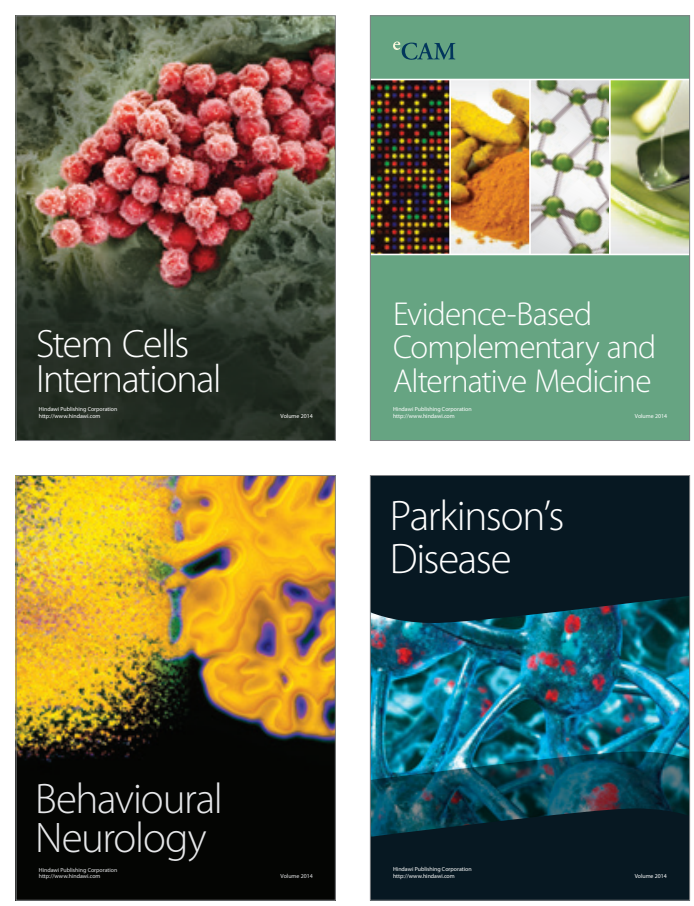

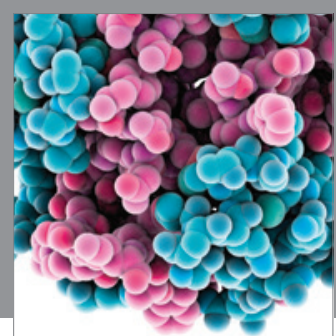

Journal of
Diabetes Research

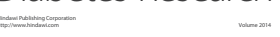

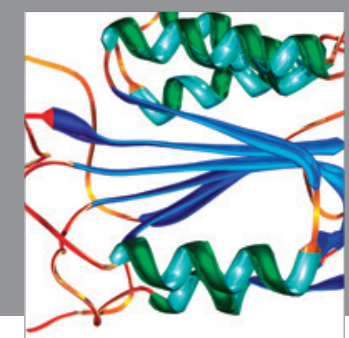

Disease Markers
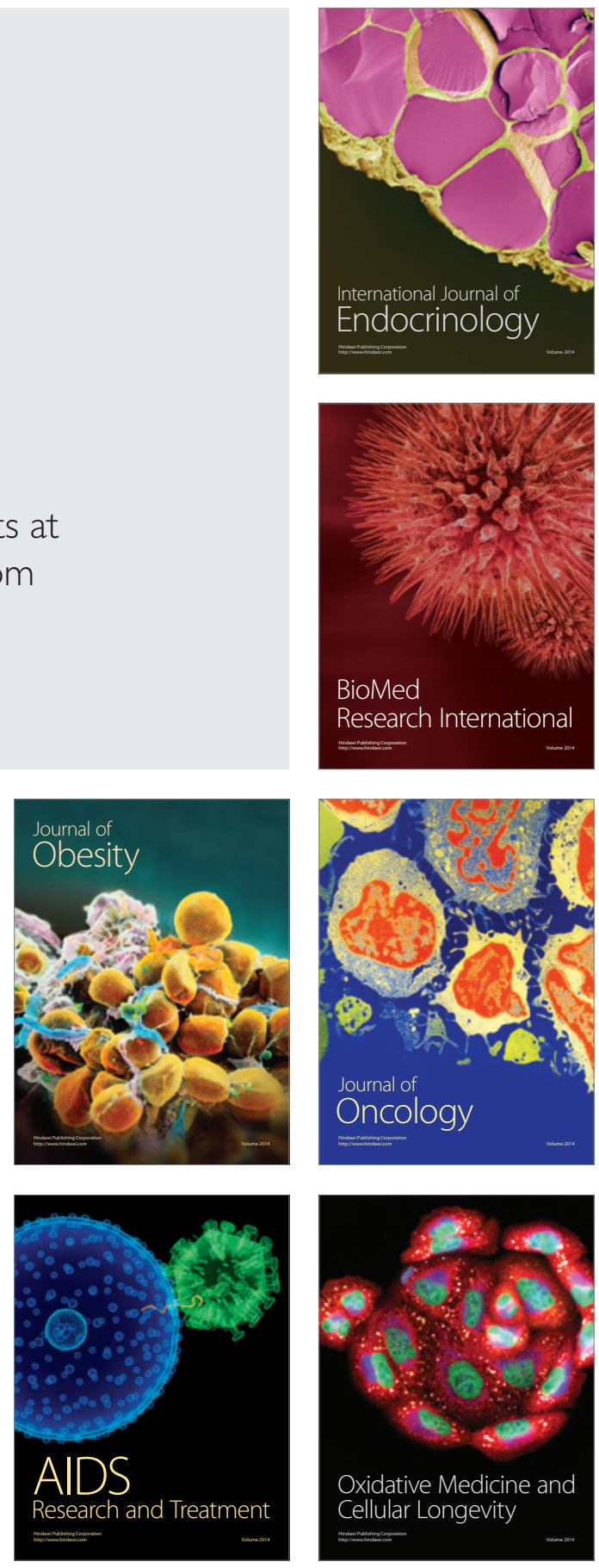